\title{
Traditional Color Image Enhancement Based on Adaptive Filter
}

\author{
Sk.Khwaja Moinuddin ${ }^{1}$, Ch.Madhuri Devi ${ }^{2}$ \\ ' Sri Indu College of Engineering \& Technology, Hyderabad \\ 2 \\ Sri Indu College of Engineering \& Technology, Hyderabad
}

\begin{abstract}
Color image enhancement is a very important pre-processing stage in face detection and face recognition applications especially when the environment is very dark. In this paper, a color color image enhancement with the adaptive filter and discrete wavelet transform (DWT) is proposed. Discrete wavelet transform is used to improve image enhancement and stationary wavelet transform is used to reduce the halo distortion in gray scale images only and wavelet cannot work in color images. In adaptive filter technique is to reduce halo distortion in color and gray images. The adaptive filter algorithm finds the importance of color information in color image enhancement and utilizes color space conversion to obtain a much better visibility. Experimental results show that adaptive filter to reducing halo, color distortion and produce better visibility compare to DWT.
\end{abstract}

Keywords-Adaptive filter, color video enhancement, HVS, color space conversion and image fusion

\section{INTRODUCTION}

Video enhancement is very useful tool in many security and surveillance applications. In nighttime images/video are difficult to understand because they lack background context due to poor illumination. As a real life example, when you look at an image or video seen from a traffic camera posted on the web or shown on TV, it is very difficult to understand from which part of the town this image is taken, how many lanes the highway has or what buildings are nearby [1]. INDANE is a more robust technique that enhances images taken under non-uniform lighting conditions by enhancing the darker regions in the image retaining the brighter regions unaffected and restoring natural colors [2]. Retinex [3-5] is an effective technique for color image enhancement, which can produce a very good enhanced result. But the enhanced image has color distortion and the calculation is complex. Li Tao and Vijayan $\mathrm{K}$.
Asari proposed a robust color image enhancement algorithm [6]. The algorithm can enhance color image without distortion, but the edges of the color image could not be handled well. The algorithm use Gaussian filter to estimate background image. Gaussian kernel function is isotropic, which leads to the inaccurate estimation of background image, resulting in the halo phenomenon. Considering the above two algorithms, a new bio-inspired color image enhancement algorithm is proposed by the author [7]. A novel algorithm based on I luminanceReflectance Model for Enhancement (IRME) has been developed and proven to be very effective for images captured under insufficient or non-uniform lighting conditions [8]. The algorithm is based on luminance perception and processing to achieve dynamic range compression while retaining or enhancing visually important features. Conventional image enhancement techniques such as global brightness and contrast enhancement, gamma compression and histogram equalization, are incapable of providing satisfactory enhancement results for underexposed or saturated images. The acquiring of the background image is important in many color image enhancement technologies and we also need to estimate the background image in this algorithm. In traditional algorithms, only distance and luminance information of pixels is considered in estimation of background image. They all overlook the important information of color image - color information.

In this paper is organized as follows. Image enhancement based on DWT in section II. Section III describes the Adaptive filter of color image. The simulation results are presented in Section IV. Concluding remarks are made in Section V.

\section{II.IMAGE ENHANCEMENT BASED ON DWT}

In this work, DWT has been employed in order to preserve the high frequency components of the image. The redundancy and shift invariance of the 
DWT mean that DWT coefficients are inherently interposable.

The interpolated high frequency sub bands and the SWT high frequency sub bands have the same size which means they can be added with each other. The new corrected high frequency sub bands can be interpolated further for higher enlargement. Also it is known that in the wavelet domain, the low resolution image is obtained by low pass filtering of the high resolution image. In other words, low frequency sub band is the low resolution of the original image. Therefore, instead of using low frequency sub band, which contains less information than the original high resolution image, we are using the input image for the interpolation of low frequency sub band image. Using input image instead of low frequency sub band increases the quality of the super resolved image

By interpolating input image by $\sigma / 2$, and high frequency sub bands by 2 and $\sigma$ in the intermediate and final interpolation stages respectively, and then by applying IDWT.

\section{COLOR IMAGE ENHANCEMENT USING} ADAPTIVE FiLTER

In this section, image enhancement technique that is based on logarithm transformation of the luminance of the pixels in the image. The algorithm consists of independent steps for luminance enhancement with dynamic range compression and contrast enhancement. The luminance enhancement step considers the maximum color component of the pixels in the nonlinear transformation with dynamic range compression based on a logarithmic approach while the ratios of the original color bands $(R, G$ and B) are preserved. The proposed steps as follows

1) Obtain Luminance And Background Image From Video

2) Adaptive adjustment

3) Color restoration

1) Obtain Luminance and Background Image From Video

First we read the input video in to the computer. Then extracted an image from the video from the background purpose which Will be Used in the processing the other frames in a video.As the camera is stationary, the background changes little during the video capture time. Oppositely, the motion part changes all the time. So we add the frames together to strengthen the background and at the same time weaken the motion part. For each frame from video to obtain luminance and background image as shown below.

The luminance image of each frame is $I_{L}(x, y)$. Subjective luminance is the logarithmic function of the light intensity into human eyes [9]. We get the logarithmic function of the original luminance image and then normalize it to get the subjective luminance $\mathrm{I}_{\mathrm{L}}$.

$$
\mathbf{I}_{\mathrm{L}}(\mathbf{x}, \mathrm{y})=\log (\mathrm{Y}(\mathrm{x}, \mathrm{y})) / \log (255)
$$

Where

$\mathrm{Y}(\mathrm{x}, \mathrm{y})=$ Brightness image in YUV color image

The color images we usually see are mostly in RGB color space, which employ red, green, and blue three primary colors to produce other colors. In RGB color pace, other colors are synthesized by three primary colors, which is not effective in some cases. Consequently, we use another color space-YUV color space instead of the RGB color space in the algorithm proposed. The importance of using YUV color space is that its brightness image $\mathrm{Y}$ and chroma images $\mathrm{U}, \mathrm{V}$ are separate. $\mathrm{Y}$ stands or the luminance, and $\mathrm{U}, \mathrm{V}$ are color components.

In this paper, to obtain the background image according to the $\mathrm{Y}, \mathrm{U}, \mathrm{V}$ values at pixel $(\mathrm{x}, \mathrm{y})$

$$
I_{B}(x, y)=\frac{\sum_{i, j=-W}^{W} G_{R} G_{I} G_{C} I\left(x_{i}, y_{j}\right)}{\sum_{i, j=-W}^{W} G_{R} G_{I} G_{C}}
$$

Where

$\mathrm{G}_{\mathrm{R}}$ is the distance parameter of intensity image. We use the below formula to obtain the distance parameter

$$
G_{R}\left(x, y, x_{i}, y_{j}\right)=\exp \left\{-\frac{\left(x-x_{i}\right)^{2}+\left(y-y_{j}\right)^{2}}{2 \sigma_{R}^{2}}\right\}
$$

Here

$(\mathrm{Xi}, \mathrm{Yi})$ is the neighbor pixels of $\mathrm{Y}, \mathrm{U}$, and $\mathrm{V}$ values

$\mathrm{G}_{\mathrm{I}}$ is the distance parameter of $\mathrm{U}, \mathrm{V}$ image. We use the below formula to obtain the distance parameter

$$
G_{I}\left(x, y ; x_{i}, y_{j}\right)=\exp \left\{-\frac{\left(I(x, y)-I\left(x_{i}, y_{j}\right)\right.}{2 \sigma_{I}^{2}}\right\}
$$

Gc is the scale parameter of pixel filtering

$$
G_{C}\left(x, y, x_{i}, y_{j}\right)=\exp \left\{\frac{\left(U(x, y)-U\left(x_{i}, y_{j}\right)\right)^{2}+\left(V(x, y)-V\left(x_{i}, y_{j}\right)^{2}\right.}{2 \sigma_{C}^{2}}\right\}
$$


Here

$\mathrm{U}(\mathrm{x}, \mathrm{y}), \mathrm{V}(\mathrm{x}, \mathrm{y})=$ chrome images of YUV image

$\mathrm{I}(\mathrm{x}, \mathrm{y})=$ intensity value at $(\mathrm{x}, \mathrm{y})$

$\sigma_{\mathrm{R}}, \sigma_{\mathrm{I}}, \sigma_{\mathrm{C}}$ are the scale parameters, whose values are 20,30,60 respectively.

Transforming the RGB color image into YUV color space, we can get directly the luminance image. Let the YUV color image through the adaptive filter, and the background image can be obtained then go for adaptive adjustment as explained in section IV.

\section{2) Adaptive Adjustment}

The image human eye seeing is related to the contrast between the image and its background image [9].By using adaptive adjustment to obtain the local enhancement $\mathrm{I}_{\mathrm{E}}(\mathrm{x}, \mathrm{y})$.

We use the formula to obtain the local enhancement

$\mathbf{I}_{\mathbf{E}}(\mathbf{x}, \mathbf{y})=\boldsymbol{\beta}(\mathbf{x}, \mathbf{y}) \cdot \mathbf{I}_{\mathbf{L}}(\mathbf{x}, \mathbf{y})$

$\boldsymbol{\beta}(\mathrm{x}, \mathrm{y})$ is the function of adaptive regulation. $\mathrm{I}_{\mathrm{E}}(\mathrm{x}$, y) is local enhanced color image, and the enhanced color image can be obtained after the color restoration for $\mathrm{I}_{\mathrm{E}}$.

Where

$\beta(x, y)=(a \alpha+b) . w(x, y) ;$

where, $\alpha$ is intensity coefficient according to the cumulative distribution function (CDF) of the luminance image. $\mathrm{W}(\mathrm{x}, \mathrm{y})$ is the ratio value between the background image and the intensity image. A and $\mathrm{b}$ are constants, we can adjust them to achieve good adjustment results.

$$
\alpha=\left\{\begin{array}{cc}
0, & g \leq 60 \\
(g-60) / 130, & 60<g \leq 190 \\
1, & g>190
\end{array}\right.
$$

$\mathrm{g}$ is the grayscale level when the cumulative distribution function(CDF) of the intensity image is 0.1 . If more than $90 \%$ of all pixels have intensity higher than $190, \alpha$ is 1 ; when $10 \%$ of all pixels have intensity lower that $60, \alpha$ is 0 ; other times $\alpha$ linear changes between 0 and 1 .

$$
\mathbf{W}(\mathbf{x}, \mathbf{y})=\mathbf{I}_{\mathbf{B}}(\mathbf{x}, \mathbf{y}) / \mathbf{I}(\mathbf{x}, \mathbf{y}) \text {; }
$$

To apply the fast Fourier transform (FFT) of $\mathrm{I}_{\mathrm{E}}$ we can get the image I'.

$$
\begin{aligned}
& R^{\prime}(x, y)=R(x, y) \frac{I^{\prime}(x, y)}{I(x, y)} \\
& G^{\prime}(x, y)=G(x, y) \frac{I^{\prime}(x, y)}{I(x, y)} \\
& B^{\prime}(x, y)=B(x, y) \frac{I^{\prime}(x, y)}{I(x, y)}
\end{aligned}
$$

\section{RESULTS}

In this section, we discuss the results of the image enhancement based on filter and wavelet. The DWT improve the visibility on gray images only as show in fig.1.The filter finds the importance of color information in color image enhancement and utilizes color space conversion to obtain a much better visibility.
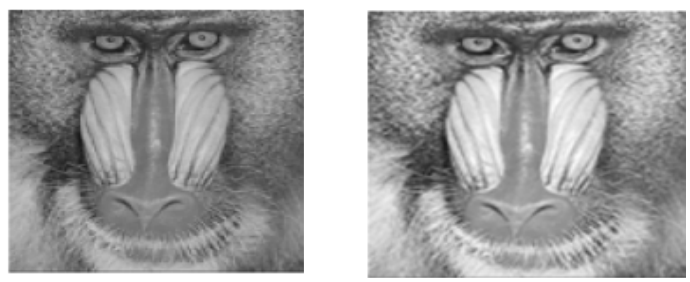

Figure 1. (a) Original low resolution Baboon's image. (d) Image enhancement using DWT
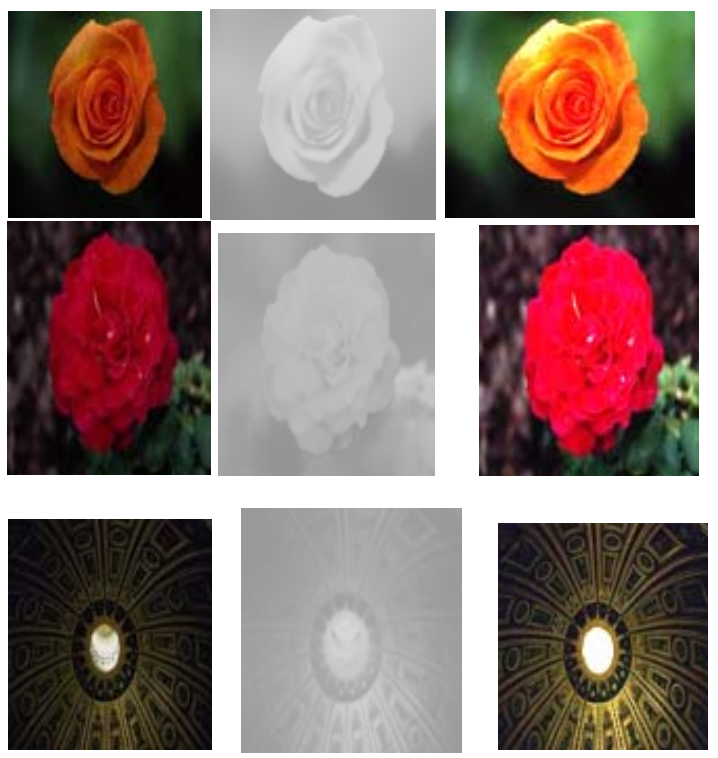

Figure 2. First colom represent as orginal frame fro video.Second colom represent as the background image o and third colom represent as enchanced images of proposed algorithm.

\section{3) Calculate Color Restoration}




\section{CONCLUSION}

A new color video enhancement algorithm is proposed in this paper. The algorithm is related to human visual It proposes a new adaptive filter has better visibility, the details are clear, and the colors are vivid and natural.

\section{REFERENCES}

[1] Ramesh Raskar, Adrian Ilie, Jingyi Yu "Image Fusion for Context Enhancement".

[2] L. Tao and K. V. Asari, "An Integrated Neighborhood Dependent Approach for Nonlinear Enhancement of Color Images," IEEE Computer Society International Conference on Information Technology: Coding and Computing ITCC 2004, Las Vegas, Nevada, April 5-7, 2004.

[3] Meylan L, Susstrunk S. High dynamic range image rendering with a retinex-based adaptive filter [J]. IEEE Transactions on Image Processing, 2006, 15(9): 2820-2830.

[4] Funt B, Ciurea F, McCann J. Retinex in MATLAB [J]. Journal of Electronic Imaging, 2004, 13(1): 48-57.

[5] Kimmel R, Elad M, Shaked D, et al. A variational framework for Retinex[J]. International Journal of Computer Vision, 2003, 52(1): 7-23.

[6] Wang Shou-jue, Ding Xing-hao, Liao Ying-hao, Guo dong-hui, A Novel Bio-inspired Algorithm for Color Image Enhancement, Acta Electronica Sinica, 2008.10, Vol.36, No.10: 1970-1973.(in Chinese)

[7] Tao, L. and Asari, K. V., "An efficient luminancereflectance nonlinear video stream enhancement model," IS\&T/SPIE Symp. On Elect. Imaging: RealTime Image Processing III, San Jose, CA, January 15-19, 2006.

[8] Webster M A. Human colour perception and its adaptation [J]. Network: Computation in Neural Systems, 1996, 7(4): 587-634. 